\title{
MADAME DE STAËL ET LA « RELIGION UNIVERSELLE »
}

L'idée de religion universelle a une longue histoire, plongeant ses racines dans l'antiquité, aussi bien païenne que chrétienne. L'évolution de l'idée accélère dans les temps modernes : c'est alors, et tout spécialement au XVIII ${ }^{\mathrm{e}}$ siècle, que se développent ses différentes variantes : apologétique et catholique, ésotérique, naturelle et philosophique (révélée et non révélée), naturaliste et astronomique. Ces différentes manifestations trouvent leur continuation au XIX ${ }^{\mathrm{e}}$ siècle dans de nouveaux contextes littéraires et socio-culturels définis par les courants du romantisme, du socialisme ou du positivisme. L'idée de religion universelle peut être formulée de façon implicite ou explicite. Ces derniers cas, qui ne sont pas rares, méritent une attention particulière dans la mesure où, la présence de l'idée n'y étant pas douteuse, ils permettent de mieux saisir le mouvement de son articulation. Le présent article se propose d'étudier la « religion universelle» telle qu'elle est conçue par Madame de Staël en 1813 dans son ouvrage De l'Allemagne. À cette fin, nous allons nous pencher sur l'identité religieuse de l'écrivaine, puis esquisser le contexte philosophico-religieux dans lequel l'idée qui nous intéresse s'est développée, ainsi que la vision du monde staëlienne, avant de voir plus en détail le sens que la pionnière du romantisme en France donne à sa « religion universelle $»$.

\section{L’IDENTITÉ RELIGIEUSE DE MADAME DE STAËL}

Germaine de Staël est originaire d'une famille protestante genevoise, dont la religiosité du côté de la mère (Suzanne Curchod) est basée sur une éducation 
morale et culturelle soignée, et du côté du père (Jacques Necker), se résume dans une version libérale du calvinisme qui avoisine la religion naturelle ${ }^{1}$. Dans les deux cas, le respect de l'autorité et de la tradition ecclésiastique se trouve complété par un intérêt profond pour la philosophie des Lumières (la mère de Madame de Staël anime un salon qui rassemble des personnalités parmi les plus renommées $\mathrm{du}$ XVIII ${ }^{\mathrm{e}}$ siècle), ce qui introduit une certaine souplesse par rapport à la doctrine chrétienne exprimée dans le credo.

Après la Révolution, Germaine de Staël, qui fréquente le salon de sa mère et prend connaissance de la pensée des Lumières, adopte une philosophie inspirée du théisme de Rousseau, et si le christianisme y occupe une place particulière, c'est seulement en tant qu'expression la plus parfaite de la religion naturelle, libre de tout dogmatisme et ritualisme. Culte évangélique du cœur, source de bonté et de consolation, cette religion se trouve inscrite dans la conscience humaine. Madame de Staël va cependant évoluer vers une spiritualité approfondie, alimentée par diverses sources mystiques et ésotériques, avec lesquelles elle se familiarise aux côtés de son mari, le baron suédois de Staël-Holstein, franc-maçon versé dans l'ésotérisme et fréquentant les cercles d'illuminés ${ }^{2}$.

Même si Madame de Staël ne fera sienne aucune doctrine ésotérique et n'adhérera jamais à aucune école ni société secrète, qui foisonnent alors en Europe, son intérêt pour la problématique mystique et théosophique - elle sait d'ailleurs parfaitement distinguer l'une de l'autre - est manifeste ${ }^{3}$. Le cercle de Coppet, qui rassemble les plus grands écrivains européens, tels que les frères Schlegel, Sismondi, Juliane de Krüdener, Zacharias Werner ou Benjamin Constant ${ }^{4}$, constitue un laboratoire de pensée où s'entrecroisent différentes influences, notamment religieuses. Coppet a aussi sa période d'intérêt pour le mysticisme et l'ésotérisme, et Bonstetten écrit à son amie en 1808 : « Rien n'est plus changé que Coppet. Tu verras, ces gens deviendront catholiques, boehmistes, martinistes, mystiques $»^{5}$.

Le voyage de Madame de Staël dans les années 1803-1804 en compagnie de Constant lui fait découvrir la nouvelle pensée allemande, pas uniquement l'idéalisme de Kant, Schelling ou Fichte, mais aussi la théosophie de Jacob Boehme et de Louis-Claude de Saint-Martin, traducteur du cordonnier de Görlitz en français,

${ }^{1}$ H. Perrochon, «Les sources suisses de la religion de $\mathrm{M}^{\mathrm{me}}$ de Staël », [dans :] Madame de Staël et l'Europe. Colloque de Coppet, 18-24 juillet 1966, Klincksieck, Paris 1970, pp. 145-149.

2 Ibidem, p. 148.

${ }^{3}$ A. Viatte, Les sources occultes du romantisme : illuminisme - théosophie : 1770-1820, t. 2, Librairie H. Champion, Paris 1969, pp. 96-131.

${ }^{4}$ Sur le cercle de Coppet, voir : E. Hofmann, F. Rosset, Le groupe de Coppet: une constellation d'intellectuels européens, Presses polytechniques et universitaires romandes, Lausanne 2005; S. Balayé, «Le groupe de Coppet : conscience d'une mission commune », [dans :] Le Groupe de Coppet : actes et documents du $2^{e}$ Colloque de Coppet, 10-13 juillet 1974, S. Balayé, J.-D. Candaux (dir.), M. Slatkine-H. Champion, Genève-Paris 1977, pp. 29-45.

${ }^{5}$ N. Jacques-Chaquin, S. Michaud, « Saint-Martin dans le groupe de Coppet et le cercle de Frédéric Schlegel », [dans :] Le Groupe de Coppet : actes et documents..., p. 117. 
qui constitue une source d'inspiration pour Franz von Baader ou Gotthilf Heinrich von Schubert. Bien que Madame de Staël acquière certaines connaissances dans le domaine, qu'elle popularisera en France dans son ouvrage De l'Allemagne (1813/1814), il est difficile d'en mesurer l'étendue. Ces connaissances semblent parfois puisées de seconde main, assimilées de façon incomplète ou inexacte ${ }^{6}$. Cela est visible sur l'exemple du cercle de Coppet, où les recherches théosophiques, qui s'expriment surtout dans l'intérêt pour la pensée de Saint-Martin, le Philosophe inconnu, se trouvent reléguées au second plan ou assujetties à d'autres courants, principalement le piétisme ou le quiétisme de Madame Guyon, propagé notamment par le chevalier de Langallerie ${ }^{7}$.

Quoi qu'il en soit, les recherches au sein du groupe de Coppet ainsi que les voyages de Madame de Staël ont un impact décisif sur sa religiosité et enrichiront le rationalisme de sa jeunesse et son protestantisme familial de nouvelles inspirations et expériences. Mais alors que chez son compagnon Benjamin Constant domine la réflexion intellectuelle sur la religion, chez Madame de Staël, c'est l'enthousiasme spirituel et poétique qui prennent le dessus. Dans De l'Allemagne, elle parle de « cette religion universelle, dont son cœur [celui de l'homme] est le sanctuaire $»^{8}$. En même temps, elle ne veut pas se faire d'illusions : si la religion l'intéresse au plus haut degré comme questionnement existentiel et tentative de comprendre la condition humaine, Madame de Staël confesse dans une lettre adressée à son cousin: " J'ai avant tout peur d'un air d'hypocrisie, et comme je mène une vie dissipée, je ne puis parler religion que comme un vœu ${ }^{9}$. Bien que la vie de Madame de Staël, et surtout ses dernières années, où elle se tourne vers une religiosité plus traditionnelle, puisse mettre en question cet aveu, il témoigne toutefois sûrement de l'inquiétude de l'écrivaine et de l'ambiguïté de son vécu du point de vue religieux. Élevée dans la tradition protestante (qu'elle transmet à ses enfants), mais aussi dans celle des Lumières, Madame de Staël se refuse à définir et clore son identité. Tout en s'inspirant de multiples courants philosophiques et ésotériques, elle a conscience du fait que l'expérience intime devance toute forme de foi ou de croyance. Et en rejetant l'intolérance et le fanatisme, elle recherche l'essence même de la religiosité : tout d'abord dans une religion naturelle socialement et politiquement utile ${ }^{10}$, puis dans une religion du cœur universelle aux résonances mystiques.

${ }^{6}$ On a souvent reproché à Madame de Staël sa connaissance étriquée des sources de sa pensée. Mais peut-on exiger d'une écrivaine voulant employer son talent à populariser certaines idées, des connaissances exhaustives sur des sujets spécialisés et complexes ?

${ }^{7}$ N. Jacques-Chaquin, S. Michaud, op. cit., pp. 118-122.

${ }^{8}$ Madame de Staël, De l'Allemagne, t. 2, Garnier-Flammarion, Paris 1968, p. 239.

${ }^{9}$ H. Perrochon, op. cit., p. 150.

${ }^{10}$ G. Gengembre, J. Goldzink, « Madame de Staël ou pour une religion politique », [dans :], Le groupe de Coppet et le monde moderne : conceptions - images — débats : actes du VI Colloque de Coppet, Liège, 10-12 juillet 1997, Bibliothèque de la Faculté de philosophie et lettres de l'Université de Liège-Droz, Liège-Genève 1998, pp. 207-222. 


\section{DES SECTES EN ALLEMAGNE : ASPECTS DU CONTEXTE PHILOSOPHICO-RELIGIEUX}

Nous comptons nous occuper dans le présent article de cette dernière idée, en nous concentrant sur certains chapitres du livre De l'Allemagne, dont la troisième partie est consacrée à la philosophie et à la morale, et la quatrième, à la religion. C'est là que nous trouvons le chapitre sur « l'esprit de secte en Allemagne " ${ }^{11}$, le mot secte désignant dans ce contexte un groupe ou une école (philosophique ou religieuse) représentant un point de vue particulier. Le chapitre donne donc un aperçu des différents mouvements philosophiques, religieux ou para-religieux présents en Allemagne vers la fin du XVIII ${ }^{\mathrm{e}}$ et au début du XIX ${ }^{\mathrm{e}}$ siècle, et son étude est extrêmement utile avant d'aborder le sujet de la « religion universelle » staëlienne, car il permet de cerner certains aspects fondamentaux du contexte historique et philosophico-religieux de sa formulation.

La spécificité des sectes allemandes se traduit dans leur poursuite désintéressée de la vérité, tendance opposée à l'activisme dominant en France, orienté sur la transformation de la société et l'exercice d'une influence sur les masses. Madame de Staël divise les « sectes » en trois catégories. Premièrement, certaines confessions issues de la Réforme, notamment les anabaptistes, qui ont vite disparu du fait de leur engagement politique révolutionnaire, ou les frères moraves, qui contrairement à eux, se retirent complètement du monde en cultivant une foi épurée, ce qui les rapproche des quakers. Deuxièmement, les sociétés secrètes, regroupant des gens aux aspirations profondes qui se reconnaissent entre eux à l'aide de certains signes pour se distinguer des autres. Elles possèdent une longue tradition, renvoyant aux mystères d'Éleusis ou aux esséniens, mais la plus ancienne parmi elles est la franc-maçonnerie. Madame de Staël expose brièvement ses principes de base, à savoir les idéaux de l'époque des Lumières, à commencer par l'indépendance d'esprit qui caractérise en général les sociétés secrètes. En se référant aux écrits maçonniques de Gotthold Ephraim Lessing ${ }^{12}$, elle évoque aussi la fraternité qui permet de dépasser les différentes barrières sociales. En Écosse et en Allemagne, où se trouvent ses origines, la franc-maçonnerie est selon Madame de Staël une institution plus « sérieuse » qu'en France, et c'est en Écosse qu'on a parlé de ses rapports avec l'ordre du Temple. Il faut ajouter ici que l'idée d'un lien de continuité historique entre francs-maçons et templiers a été rejetée au convent maçonnique de Wilhelmsbad, évoqué d'ailleurs par Madame de Staël. Le convent, qui a lieu en 1782 sous la présidence du duc Ferdinand de Brunswick, était dominé par les francs-maçons mystiques et les martinistes lyonnais : Jean-Baptiste Willermoz créera à cette occasion le Rite écossais rectifié, fondé sur la doctrine de

11 Madame de Staël, op. cit., pp. 285-290.

12 Il s'agit de Ernst et Falk: causeries pour francs-maçons, terminées en 1780, date de parution de L'éducation du genre humain. Lessing, bibliothécaire chez le duc Charles-Guillaume-Ferdinand de Brunswick-Wolfenbüttel, est initié à la loge Aux Trois Roses d'Or à Hambourg en 1771. 
Martinès de Pasqually ${ }^{13}$. Madame de Staël n'est donc pas tout à fait juste en affirmant que ce sont les enseignements de Saint-Martin qui ont marqué la réunion.

Enfin, la troisième catégorie de secte citée est celle des illuminés, que l'écrivaine divise en mystiques, politiques et visionnaires ${ }^{14}$. Aux premiers appartiennent Jacob Boehme, Saint-Martin et Pasquallis [sic]. Ils se considèrent comme membres de l'Église intérieure, s'intéressent surtout aux questions religieuses et à l'interprétation de la nature par le prisme des vérités de la foi. En parlant des illuminés politiques, Madame de Staël pense aux illuminés de Bavière dirigés par Weishaupt, et elle est loin d'être élogieuse à leur égard. Ils s'unissent autour des mêmes convictions et objectifs, mais sont surtout liés par des intérêts communs : la pratique du secret leur permet de gagner le contrôle sur les gens et d'en tirer des profits. Ce jugement n'a rien d'étonnant, les illuminés de Bavière, purement rationalistes, ne jouissant pas alors d'une bonne réputation parmi les personnes favorables à la religion et les penseurs spiritualistes. Si la franc-maçonnerie est longtemps accusée de complot visant la destruction de la monarchie et du christianisme, c'est justement en raison d'une identification du mouvement avec les Bavarois ${ }^{15}$.

À la tête des visionnaires, Madame de Staël place Emanuel Swedenborg et souligne leur foi dans l'évocation des esprits des morts et dans le pouvoir d'opérer des miracles par la force de la volonté. Cette approche " empirique » est critiquée par les idéalistes (supposément Saint-Martin, qui n'appréciait effectivement pas Swedenborg). Il faut observer que si l'image du Suédois qui se dégage du texte de Madame de Staël est assez déformée (sa doctrine étant en réalité beaucoup plus sobre, orientée sur le développement de la sagesse et de l'amour), le swedenborgisme en France fonctionne d'habitude en association avec les pratiques du magnétisme animal ou de l'alchimie hermétique ${ }^{16}$. En ce qui concerne les recherches des alchimistes et des magnétiseurs, l'écrivaine ne les juge pas de façon négative : leur aspiration à sonder les mystères de la nature s'oppose à une vision des choses matérialiste. Ce genre d'expérimentation peut sans doute être dangereuse, mais voir dans le monde un mécanisme déterminé par des lois inébranlables est tout aussi dangereux.

Dans d'autres chapitres Madame de Staël consacre plus de place aux différentes confessions chrétiennes ainsi qu'aux «philosophes religieux appelés théosophes ${ }^{17}$. En caractérisant ceux-ci, l'écrivaine reprend une distinction qui constitue une sorte de leitmotiv dans ses réflexions : il y a les mystiques, qui se concentrent sur la

13 J. Palou, La franc-maçonnerie, Payot, Paris 1964, pp. 175-177.

${ }^{14}$ Madame de Staël, op. cit., p. 288.

15 Joseph de Maistre prendra dans ce contexte la défense des illuminés mystiques et polémiquera avec les thèses de l'abbé Barruel sur le rôle de la franc-maçonnerie dans l'éclatement de la Révolution ; voir: É. Dermenghem, Joseph de Maistre, mystique : ses rapports avec le martinisme, l'illumiminisme et la franc-maçonnerie, l'influence des doctrines mystiques et occultes sur sa pensée religieuse, La Connaissance, Paris 1923, pp. 83-104.

${ }^{16}$ Par exemple chez Pernety et les illuminés d'Avignon ; voir: K.-E. Sjöden, Swedenborg en France, Almqvist \& Wiksell, Stockholm 1985.

${ }^{17}$ Madame de Staël, op. cit., pp. 281-283. 
vie intérieure et leur rapport avec le Divin, et ceux qui se tournent vers l'extérieur afin d'étudier la nature, qui constitue une révélation permanente. C'est parmi eux que l'on trouve Boehme et Saint-Martin, qui dévoilent les lois spirituelles gouvernant le monde à l'aide de symboles puisés dans la Bible, et voient dans chaque élément de la nature le reflet des dogmes de la chute et de la régénération, ainsi que l'action des principes de la colère et de la miséricorde.

\section{LA VISION DU MONDE STAËLIENNE}

Afin de saisir l'importance qu'ont pour Madame de Staël les idées des illuminés et des théosophes comme Boehme et Saint-Martin, il faut voir plus en détail sa vision de l'homme et de l'univers. On la trouvera notamment exposée dans ses réflexions sur « l'influence de la nouvelle philosophie allemande sur les science ${ }^{18}$ ou encore sur « la contemplation de la nature $»{ }^{19}$. En parlant de « nouvelle philosophie allemande », Madame de Staël a en vue, plutôt que la philosophie idéaliste, la philosophie de la nature (Naturphilosophie) liée aux noms de Schelling, Schubert, Baader ou encore Novalis, qui se sont fortement inspirés des œuvres du Philosophe inconnu, de Boehme, de Paracelse ou des mystiques néoplatoniciens. On peut facilement constater que la vision du monde présentée par l'auteure de De l'Allemagne contient tous les éléments caractéristiques de la « forme de pensée » que constitue pour Antoine Faivre " l'ésotérisme occidental ${ }^{20}$. Rappelons que les éléments en question sont : une conception vivante de la nature, l'existence de correspondances et d'analogies en son sein, le rôle de l'imagination et de médiations symboliques, l'expérience d'une transformation intérieure, ainsi que la transmission initiatique et la recherche d'un fond commun de diverses traditions (les deux derniers étant des éléments secondaires).

Voyons de quelle façon ces idées sont présentes dans l'œuvre de Madame de Staël. Tout d'abord, conformément à la manière dont tout le romantisme va envisager la nature, l'univers est ici un organisme vivant, dans lequel tous les éléments sont liés entre eux. Les phénomènes naturels possèdent un sens philosophique et religieux qui se définit par les rapports entre la nature, l'homme et le Divin. Et même si ce sens est inépuisable, il est possible de le dévoiler et de le deviner progressivement. Tout le mystère de l'univers et de l'homme se cache dans les principes interdépendants de la vie et de la volonté, associés respectivement au monde physique et moral ${ }^{21}$. À propos des représentants de la nouvelle philosophie allemande, Madame de Staël écrit :

\footnotetext{
18 Ibidem, pp. 165-175.

${ }^{19}$ Ibidem, pp. 291-300.

${ }^{20}$ A. Faivre, L'ésotérisme, Paris 1992, pp. 18-23.

${ }^{21}$ Madame de Staël, op. cit., p. 169.
} 
Deux grandes vues générales leur servent de guide dans l'étude des sciences : l'une, que l'univers est fait sur le modèle de l'âme humaine ; et l'autre, que l'analogie de chaque partie de l'univers avec l'ensemble est telle que la même idée se réfléchit constamment du tout dans chaque partie, et de chaque partie dans le tout. [...] Chaque plante, chaque fleur, contient le système entier de l'univers; un instant de vie recèle en son sein l'éternité ; le plus faible atome est un monde, et le monde peut-être n'est qu'un atome. Chaque portion de l'univers semble un miroir où la création tout entière est représentée, et l'on ne sait ce qui inspire le plus d'admiration, ou de la pensée, toujours la même, ou de la forme, toujours diverse ${ }^{22}$.

L'unité qui règne au sein de l'univers se manifeste de multiples manières. Puisqu'il n'y a qu'un seul Créateur, il peut exprimer les mêmes vérités dans des langages différents. Autrement, comment serait-il possible d'expliquer les rapports étonnants entre les formes, les parfums, les sons et les couleurs ? Comment expliquer que, comme l'a observé Chladni, le sable, soumis à des vibrations harmonieuses ou des dissonances, forme des figures régulières ou irrégulières ? ${ }^{23}$

Lorsqu'on parle des rapports unissant l'homme et la nature, lorsqu' on associe la colère à la mer déchaînée, le calme au clair de lune ou la tristesse à un ciel nuageux, il ne s'agit pas de métaphores gratuites, mais de la structure même de l'univers, par essence symbolique ${ }^{24}$, et qui ressemble à un poème bien plus qu'à une machine ${ }^{25}$. Car c'est l'imagination qui nous rapproche de la vérité plus que les mathématiques, même si elle n'exclut pas ces dernières. L'unité du monde suppose en effet l'unité du savoir, et l'observation des faits devrait être accompagnée d'une vision synthétique intuitive. Ces différents aspects de la connaissance sont complémentaires, tout comme les diverses branches de la science, la poésie, la philosophie et la religion. Ainsi, il est difficile de maîtriser une discipline choisie sans l'étude des autres. Les Anciens avaient la raison pour guide, mais consultaient aussi l'imagination, qui est la prêtresse de la nature. Qui sait si ce qu'on considère comme des erreurs grossières et des superstitions ne renvoie pas à des lois qui n'ont pas encore été découvertes? Les rapports entre les corps célestes et les métaux, les oracles et les prophéties, n'ont-ils pas leur source dans des puissances inconnues? Et ce qui passe pour folie et aberration ne contient-il pas un germe de vérité ? Il ne s'agit donc pas de renoncer à la méthode empirique, mais de l'incorporer dans « une philosophie plus étendue, qui embrasserait l'univers dans son ensemble, et ne mépriserait pas le côté nocturne de la nature $»^{26}$. Si

${ }^{22}$ Ibidem, pp. 167-168. Le passage rappelle le fameux « tout est lié », qui est à la base de toute vision du monde ésotérique et de toute magie ou pratique occulte.

23 Ibidem, p. 168.

24 Ibidem, p. 167.

25 Ibidem, p. 174.

${ }^{26}$ Ibidem, p. 173. L'expression est utilisée par plusieurs philosophes de la nature allemands, à commencer par Schubert. L'idée qui la sous-tend fait l'objet du livre d'Albert Béguin L'âme romantique et le rêve, J. Corti, Paris 1960. Elle revient aussi chez Baudelaire dans le Salon de 1859 (chapitre "Le gouvernement de l'imagination »), où l'auteur se réfère à l'ouvrage de Catherine Crowe: The Night Side of Nature (1848) ; Ch. Baudelaire, Euvres complètes, texte établi, présenté 
Madame de Staël prend la défense des alchimistes, des illuminés et des magnétiseurs, c'est parce qu'ils tentent de déchiffrer les mystères de la nature, parfois à l'aveuglette, mais guidés par l'inspiration imaginative. Il faut donc canaliser l'énergie de leurs efforts plutôt que réprimer leurs recherches.

La répulsion de Madame de Staël pour une vision du monde purement matérialiste est due au fait que l'enjeu de chaque philosophie et de chaque conception métaphysique réside dans leurs conséquences spirituelles et morales ${ }^{27}$. En dernière instance, la seule chose qui compte est l'influence d'une opinion sur la condition de l'âme. Nous trouvons une approche similaire, mais appliquée à la religion, chez Kant, penseur qui constitue l'un des principaux points de référence pour Madame de Staël. Dans ses « considérations générales sur la religion en Allemagne », l'écrivaine constate:

[...] depuis vingt ans, depuis que les écrits de Kant ont fortement influé sur les esprits, il s'est établi dans la manière de concevoir la religion une liberté et une grandeur qui n'exigent ni ne rejettent aucune forme de culte en particulier, mais qui font des choses célestes le principe dominant de l'existence ${ }^{28}$.

\section{SENTIMENT RELIGIEUX ET RELIGION UNIVERSELLE}

Au rôle clé de Kant dans le développement des idées religieuses en Europe au seuil du XIX ${ }^{\mathrm{e}}$ siècle, il faut ajouter l'influence exercée par l'œuvre de Lessing (déjà cité plus haut), auteur de L'éducation du genre humain (1780) où il expose sa conception de l'évolution de la conscience religieuse de l'humanité :

Lessing dit, dans son Essai sur l'éducation du genre humain, que les révélations religieuses ont toujours été proportionnées aux lumières qui existaient à l'époque où ces révélations ont paru. L'Ancien Testament, l'Évangile, et, sous plusieurs rapports, la réformation, étaient, selon leur temps, parfaitement en harmonie avec les progrès des esprits ; et peut-être sommes-nous à la veille d'un développement du christianisme qui rassemblera dans un même foyer tous les rayons épars, et qui nous fera trouver dans la religion plus que le bonheur, plus que la philosophie, plus que la morale, plus que le sentiment même, puisque chacun de ces biens sera multiplié par sa réunion avec les autres ${ }^{29}$.

Bien que Madame de Staël ne l'indique pas, elle doit savoir que Lessing se réfère dans son ouvrage à Joachim de Fiore et sa vision d'un nouvel âge de l'Esprit qui accomplira le christianisme ${ }^{30}$. Cette idée d'un renouveau de la religion

et annoté par C. Pichois, Bibliothèque de la Pléiade, t. II, Gallimard, Paris 1976, pp. 623-624 ; 1393-1394 (note 3 de la page 623).

${ }^{27}$ Madame de Staël, op. cit., pp. 89-92.

${ }^{28}$ Ibidem, p. 237.

29 Ibidem, pp. 237-238.

${ }^{30}$ G.E. Lessing, Ernst et Falk : dialogues maçonniques [Gespräche für Freimaurer]. L'Éducation du genre humain [Die Erziehung des Menschengeschlechts], traduction et préface de P. Grappin, Aubier, Paris 1946, pp. 129-133. 
chrétienne fait penser à ce que l'auteur de De l'Allemagne écrit au sujet de « la tendance appelée mysticité », à laquelle elle consacre un chapitre entier ${ }^{31}$. La mysticité n'est rien d'autre pour elle qu'une certaine disposition religieuse, un amour pur envers le Créateur, « une manière plus intime de sentir et de concevoir le christianisme $\aleph^{32}$, que découvrent et prêchent les mystiques. Or, ceux-ci se divisent en deux catégories : ceux qui se limitent à l'étude du cœur et ceux qui élargissent cette étude sur le mystère de la création, à savoir les théologiens philosophes nommés aussi théosophes, mentionnés déjà à plusieurs reprises. Si le chapitre sur la mysticité se concentre davantage sur la première catégorie, où Madame de Staël place certains Pères de l'Église, Thomas à Kempis, Fénelon et François de Sales, on y trouve aussi une citation de Saint-Martin ${ }^{33}$.

À la base de la mysticité se trouve ce que Madame de Staël nomme le « sentiment religieux », notion conceptualisée une dizaine d'années plus tard par son compagnon Benjamin Constant. Le sentiment religieux est une «paix intérieure pleine de vie », une « réunion totale du mouvement et du repos » ${ }^{34}$, un état d'harmonie avec l'ordre universel contraire à notre égoïsme inné, un abandon sans réserve à la Providence indépendamment du cours des choses ${ }^{35}$. En défendant les quiétistes contre leurs accusateurs, l'auteure affirme que cet abandon n'exclut ni une attitude active devant la vie, ni le respect des règles morales. Ensuite, conformément au sens que lui donnera Constant ${ }^{36}$, le sentiment religieux ne relève pas de la sentimentalité, mais plutôt du sens religieux de l'homme, de son orientation sur ce qui transcende l'expérience et l'intérêt personnel. Il s'agit donc d'une constante de la nature humaine qui évolue avec le cours de l'histoire : «Tous les dogmes et tous les cultes sont les formes diverses que ce sentiment religieux a revêtues selon les temps et selon les pays ${ }^{37}$. En changeant de forme, le sentiment religieux devient le moteur d'un perfectionnement incessant, ainsi qu'un appel vers l'infini et l'inconnu.

${ }^{31}$ Madame de Staël, op. cit., pp. 263-272.

32 Ibidem, p. 263.

33 «Un écrivain français qui a des lueurs sublimes, M. de Saint-Martin, a dit que la prière était la respiration de l'âme» (ibidem, p. 266).

${ }^{34}$ Ibidem, p. 263.

35 Ibidem, pp. 265-266.

${ }^{36}$ Constant écrit dans son ouvrage phare sur la religion : « Il y a, nous l'avons dit, quelque chose d'indestructible dans la religion. Elle n'est ni une découverte de l'homme éclairé qui soit étrangère à l'homme ignorant, ni une erreur de l'homme ignorant dont l'homme éclairé se puisse affranchir. Mais il faut distinguer le fond d'avec les formes, et le sentiment religieux d'avec les institutions religieuses [...] tandis que le fond est toujours le même, immuable, éternel, la forme est variable et transitoire » (B. Constant, De la religion considérée dans sa source, ses formes et ses développements, Bossange père, Paris 1824-1831, t. 1, <http://gallica.bnf.fr/ark:/12148/bpt6k28037n> [consulté le 5.11.2016], pp. 25-26).

${ }^{37}$ Madame de Staël, op. cit., p. 266. 
Nous revenons ainsi aux «Considérations générales sur la religion en Allemagne », dans lesquelles Madame de Staël constate que « [c]'est au sentiment de l'infini que la plupart des écrivains allemands rapportent toutes les idées religieuses ${ }^{38}$. Dans le cas du sentiment de l'infini, tout comme dans celui du sentiment religieux - les deux termes peuvent être considérés, sinon comme synonymes, du moins comme étroitement liés - il faudrait plutôt parler du sens de l'infini, d'une certaine disposition innée de l'homme. Madame de Staël mentionne la conception de l'infini mathématique, à caractère purement négatif, mais aussi « le sentiment de l'infini, tel que l'imagination et le cœur l'éprouvent », qui est « positif et créateur », venant de l'enthousiasme suscité par le beau idéal ${ }^{39}$.

Le sentiment de l'infini se manifeste dans tout ce qui élève l'homme et l'arrache à ses calculs et son égoïsme étroit : les recherches métaphysiques, le dévouement, la vertu et la vie morale, l'art et le génie artistique, la religion et l'expérience de l'amour divin, la fascination qu'inspirent les spectacles de la nature :

Quand nous contemplons le ciel étoilé, où des étincelles de lumière sont des univers comme le nôtre, où la poussière brillante de la voie lactée trace avec des mondes une route dans le firmament, notre pensée se perd dans l'infini, notre cœur bat pour l'inconnu, pour l'immense, et nous sentons que ce n'est qu'au delà des expériences terrestres que notre véritable vie doit commencer. [...] Le sentiment de l'infini est le véritable attribut de l'âme : tout ce qui est beau dans tous les genres excite en nous l'espoir et le désir d'un avenir éternel et d'une existence sublime : on ne peut entendre ni le vent dans la forêt, ni les accords délicieux des voix humaines ; on ne peut éprouver l'enchantement de l'éloquence ou de la poésie ; enfin, surtout, on ne peut aimer avec innocence, avec profondeur, sans être pénétré de religion et d'immortalité ${ }^{40}$.

La religion, comme chez Constant, est toujours orientée sur l'infini, sur ce qui échappe à nos bornes. C'est pour cela qu'elle ne peut pas s'enfermer dans un dogmatisme rigide. Cela est en rapport étroit avec la dimension symbolique de tous les phénomènes de la nature. Le sentiment de l'infini ${ }^{41}$ n'est pas propre uniquement à l'être humain. Tout l'univers appelle en effet ce qui ne peut être nommé :

38 Ibidem, p. 238.

${ }^{39}$ Madame de Staël se rappelle sans doute ce que Kant ou Burke disent à propos du sublime. Cette catégorie esthétique est en effet en étroit rapport avec l'idée de l'infini.

${ }^{40}$ Madame de Staël, op. cit., pp. 238-239.

${ }^{41}$ Outre l'influence possible de Constant, qui évoque le sentiment religieux dès 1804, il importe de noter qu'en caractérisant le sentiment de l'infini, Madame de Staël mentionne, de façon très élogieuse, l'œuvre de Frédéric Ancillon, vraisemblablement son Essai sur l'idée et le sentiment de l'infini, publié dans Mélanges de littérature et de philosophie, t. 1, chez F. Schoell et H. Nicolle, Paris 1809, <http://gallica.bnf.fr/ark:/12148/bpt6k6567192t> [consulté le 5.11.2016], pp. 3-44. 
La nature a revêtu l'infini des divers symboles qui peuvent le faire arriver jusqu'à nous : la lumière et les ténèbres, l'orage et le silence, le plaisir et la douleur, tout inspire à l'homme cette religion universelle dont son cœur est le sanctuaire ${ }^{42}$.

La « religion universelle» dont parle ici Madame de Staël transcende tous les cultes religieux particuliers. Elle fait participer l'homme avec toutes ses facultés aux « rites saints du merveilleux univers $»^{43}$. «La religion n'est rien si elle n'est pas tout $»^{44}$ : expression qui souligne bien l'universalité de la religion et la tolérance qui en découle, telle qu'elle est conçue par les Allemands : « ce n'est point par indifférence qu'ils sont tolérants, c'est parce qu'ils ont de l'universalité dans leur manière de sentir et de concevoir la religion $»^{45}$. Il ne s'agit pas seulement d'une tolérance envers une autre foi, mais aussi envers d'autres manières de vivre la même foi, ouverte à toutes les sensibilités, ne cloisonnant pas la religion dans la sphère de la piété, mais incluant en elle chaque domaine de la vie :

Un grand nombre de pratiques sont recommandées aux fidèles, afin qu'à tous les moments du jour la religion leur soit rappelée par les obligations qu'elle impose ; mais si la vie entière pouvait être naturellement et sans efforts un culte de tous les instants, ne serait-ce pas mieux encore ? Puisque l'admiration pour le beau se rapporte toujours à la Divinité, et que l'élan même des pensées fortes nous fait remonter vers notre origine, pourquoi donc la puissance d'aimer, la poésie, la philosophie, ne seraient-elles pas les colonnes du temple de la foi $?^{46}$

Ce passage ne manque pas d'évoquer le « culte en esprit et en vérité » de l'Évangile selon Jean, expression d'une religion intériorisée et abolissant les divisions entre les cultes particuliers attachés à leurs pratiques. Les conclusions de Madame de Staël concernant la mysticité vont dans le même sens, exprimant

42 Madame de Staël, op. cit., p. 239. Constant écrira dans De la religion... : « Tous cherchaient l'origine de la religion dans des circonstances étrangères à l'homme, les dévots comme les philosophes. Les uns ne voulaient pas que l'homme pût être religieux sans une révélation particulière et locale ; les autres sans l'action des objets extérieurs. De là une erreur première, de là une série de longues erreurs. Oui, sans doute, il y a une révélation, mais cette révélation est universelle, elle est permanente, elle a sa source dans le cœur humain. L'homme n'a besoin que de s'écouter lui-même, il n'a besoin que d'écouter la nature qui lui parle par mille voix, pour être invinciblement porté à la religion. Sans doute aussi, les objets extérieurs influent sur les croyances mais ils en modifient les formes, ils ne créent pas le sentiment intérieur qui leur sert de base » (B. Constant, op. cit., pp. 15$17)$; et dans une note en bas de page, il ajoute : "Affirmer que le germe de la religion se trouve dans le cœur de l'homme, ce n'est assurément point assigner à ce don du ciel une origine purement humaine. L'être infini a déposé ce germe dans notre sein, pour nous préparer aux vérités que nous devions connaître » (ibidem, p. 14).

${ }^{43}$ Madame de Staël, op. cit., p. 241.

44 Ibidem.

45 Ibidem, p. 240. Le principe de la tolérance religieuse a été notamment mis en valeur par Lessing dans son drame Nathan le Sage (1779).

46 Ibidem, p. 242. 
l'attente du christianisme renouvelé des prophéties joachimites : « La philosophie idéaliste, le christianisme mystique, et la vraie poésie, ont, à beaucoup d'égards, le même but et la même source $»^{47}$.

\section{CONCLUSION}

Comme nous avons pu nous en convaincre, l'idée de religion universelle formulée par Madame de Staël dans De l'Allemagne possède de nombreuses sources. Nous avons affaire ici à une synthèse philosophico-religieuse de l'universalisme des Lumières avec son idée de religion naturelle, du protestantisme à la fois libéral et piétiste, de la mystique du quiétisme, ainsi que de sources illuministes ou ésotériques. Si elle n'a pas un caractère strictement ésotérique, toute la vision du monde qui sous-tend l'idée de religion universelle staëlienne est directement inspirée des œuvres des théosophes et des philosophes de la nature allemands, répondant aux critères de l'ésotérisme : l'analogie y lie tous les éléments d'une nature qui vit et souffre, que l'imagination permet de traduire en symboles débouchant sur l'infini. Cette conception porte en soi l'intuition d'un culte qui embrasse tout l'univers et a son temple dans le cœur humain, au-delà de toute différence entre religions instituées. Par ailleurs, les réflexions sur les sociétés secrètes et les cultes à mystères se rapportent à l'imaginaire de l'initiation : la figure de Novalis, initié tout comme ses Disciples de Saïs ${ }^{48}$, incarne la magie de l'imagination qui génère le monde et permet de sonder ses mystères. Aussi bien l'idée de religion universelle que celle de l'initiation constituent pour Madame de Staël, comme pour d'autres romantiques, des images hautement suggestives qui renvoient, pour employer les termes de Georges Gusdorf, à une surabondance de sens ${ }^{49}$. La " religion universelle » staëlienne porte aussi l'empreinte des idées religieuses de Constant et de Lessing, annonciatrices du rôle que le principe de perfectibilité va jouer tout au long du XIX ${ }^{\mathrm{e}}$ siècle, donnant lieu à des développements de l'idée de religion universelle comme ceux de Ballanche ou Leroux. Dans l'évolution de l'idée qui nous intéresse, l'exemplification originale qu'en fournit Madame de Staël occupe une place importante, assurant la transition du XVIII ${ }^{\mathrm{e}}$ au $\mathrm{XIX}^{\mathrm{e}}$ siècle, et du crépuscule des Lumières à l'aube du romantisme.

47 Ibidem, p. 272.

48 Ibidem, p. 293.

${ }^{49}$ G. Gusdorf, Le Romantisme, t. II : L'homme et la nature, Éditions Payot \& Rivages, Paris 1993, p. 117. 


\section{MADAME DE STAËL AND THE “UNIVERSAL RELIGION”}

\section{Summary}

The object of the article is the idea of a universal religion formulated by Madame de Staël in her book De l'Allemagne in 1813. In order to grasp the meaning of this idea, we have to consider first the religious identity of the author, then sketch the philosophical and religious context in which the idea has developed, and present Madame de Staël's worldview, inspired by Enlightenment philosophy and Protestant thought as well as mystical and esoteric sources popular among German philosophers of nature. In the broader context of the development of the idea of a universal religion, the work of the French pioneer of Romanticism, in which the idea, associated with the feeling of infinity, is conceived as a worship exercised by the whole universe having his temple in the human heart, occupies an important place at the intersection of the $18^{\text {th }}$ and the $19^{\text {th }}$ century.

Key words: Madame de Staël, universal religion, history of ideas, philosophy of religion, western esotericism, romantic worldview, Germany. 